\section{Tratamiento ortodóncico de camuflaje de una maloclusión de Clase III. Reporte de caso}

\author{
Orthodontic camouflage treatment of Class \\ III malocclusion. Case report
}

\begin{abstract}
Resumen
Desde el punto de vista ortodóncico y maxilofacial, una maloclusión con desarmonía esqueletal compleja, puede ser abordada con un enfoque ortopédico, ortodóncico y/o ortoquirúrgico; sin embargo, en pacientes adultos que rechazan la opción quirúrgica, la ortodoncia de camuflaje es la única opción disponible. Este artículo reporta el tratamiento de camuflaje ortodóncico de un paciente masculino de 21 ańos y 2 meses con un patrón esquelético de Clase III ángulo bajo, mordida bis a bis y tercio facial inferior disminuido. El uso de un arco utilitario de protrusión, bloques de acrílico posterior, elásticos intermaxilares de clase III, arco multiloops y stripping inferior fueron utilizados. Después de 26 meses de tratamiento, se logró una relación molar y canina de Clase I, overbite y overjet funcional, reducción de la proyección del labio inferior y mayor exposición de incisivos superiores en sonrisa con un perfil facial más agradable. Se realizó un control de 11 meses postratamiento revelando estabilidad de los resultados obtenidos.
\end{abstract}

Palabras clave: Cirugía ortognática; Maloclusión de Angle Clase III; Ortodoncia.

\section{Caso Clínico}

Loidy Liceth Ypanaque Ramirez ${ }^{1, a}$, Hugo Javier Luque Luque ${ }^{1, b}$

${ }^{1}$ Universidad Nacional Mayor de San Marcos Facultad de Odontología. Lima, Perú.

a Cirujano Dentista.

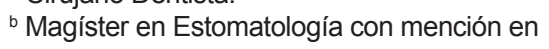
Ortodoncia.

\section{Correspondencia:}

Loidy Liceth Ypanaque Ramirez

Correo electrónico: llyr_94@hotmail.com

Av. Juan Lecaros 171, Puente Piedra, Lima, Perú.

Coautor:

Hugo Javier Luque Luque hjl150@hotmail.com

Conflicto de intereses: Los autores declaran no tener conflicto de interés.

Fuente de financiamiento: Autofinanciado.

Fecha de recepción: 01/05/18

Fecha de aceptación: 15/08/18

\begin{abstract}
From the orthodontic and maxillofacial point of view, a malocclusion with a severe skeletal disharmony can be treated with an orthopedic, orthodontic and/or orthognathic approach; however, some adult patients reject the surgical option, in those cases, orthodontic camouflage might be the only alternative. This article reports the orthodontic camouflage of a 21 year and 2 months old male patient with a Class III low angle skeletal pattern, edge to edge anterior bite relationship and decresead lower facial third. A modified utility arch, posterior acrylic bite block, Class III intermaxillary elastics, multiloop archwire and lower interproximal reduction were used. After 26 months of treatment, Class I molar and canine relationship with functional overbite and overjet was achieved. In addition, reduction of lower lip protrusion, greater exposure of upper incisors at smile and a pleasant facial profile was obtained. 11 months follow up reveals stability of the results.
\end{abstract}

Keywords: Malocclusion; Angle Class III; Orthodontics; Orthognathic surgery. 


\section{Introducción}

El desarrollo de la maloclusión esquelética Clase III constituye uno de los retos más difíciles al que se puede enfrentar un ortodoncista, especialmente si el tratamiento se basa sólo desde el punto de vista ortodóncico ${ }^{1,2}$. Su prevalencia varía grandemente entre grupos raciales y etcnicos ${ }^{3}$, siendo en asiáticos el $12 \%$, europeos $1,5-5,3 \%$, y caucásicos $1-4 \%{ }^{4-8}$.

Existen tres principales opciones de tratamiento para corregir una maloclusión de Clase III: la redirección de crecimiento, la cirugía ortognática combinada con un tratamiento ortodóncico y la terapia ortodóncica de camuflaje ${ }^{1,6}$. La redirección de crecimiento maxilofacial de Clase III, a través de aparatos ortopédicos, es una buena opción en niños que presentan un buen potencial de crecimiento. La cirugía ortognática más tratamiento ortodóncico en adultos debe ser la alternativa de primera elección en casos de mayor complejidad para la corrección de la discrepancia maxilomandibular y el desbalance de las proporciones faciales ${ }^{9,10}$. La ortodoncia de camuflaje es una opción de tratamiento para los pacientes adultos que rechazan la opción quirúrgica; sin embargo, son realizadas en casos de ligera a moderada complejidad con una estética facial aceptable ${ }^{3}$.

En nuestro medio, pocos pacientes aceptan la opción quirúrgica debido al posible riesgo y alto costo que esto conlleva, así como de otros factores. El objetivo de un tratamiento ortodóncico de camuflaje es obtener una aceptable oclusión funcional y estética a través de una compensación dentoalveolar; es decir, el desplazamiento relativo de los dientes en relación a su hueso de soporte $^{3,11,12}$.

La estrategia para un camuflaje de Clase III usualmente incluye la proinclinación de incisivos superiores, retroinclinación de incisivos inferiores, distalización de molares inferiores, mesialización de molares superiores, ambas asociadas a un tratamiento con o sin extracciones; no obstante, los cambios esqueléticos y tejidos blandos son bajos ${ }^{7}$.

Kerr et al. sugirieron que la cirugía ortognática en pacientes adultos con maloclusión de Clase III debe ser realizada en pacientes con un ángulo ANB menor de $-4^{\circ}{ }^{13}$. Stellzig-Eisenhauer et al. desarrollaron una fórmula en base a cuatro parámetros para determinar si los pacientes con Clase III podrían ser sometidos a tratamiento de ortodoncia o cirugía ortognática; estos parámetros fueron: índice de Wits, longitud anterior de la base craneal anterior, relación maxila/mandibular, y ángulo gonial inferior ${ }^{14}$. Sin embargo, ambos estudios no probaron ser específicos para distinguir entre un tratamiento ortoquirúrgico u ortodóncico de camuflaje en pacientes de maloclusión de Clase III borderline. El presente artículo reporta el caso clínico de un paciente tratado con aparatología fija ortodóncica de camuflaje en una maloclusión de Clase III.

\section{Reporte del caso}

Diagnóstico. Paciente masculino de 21 años 2 meses acudió a la Clínica de Posgrado de la Facultad de Odontología de la Universidad Nacional Mayor de San Marcos (UNMSM) Lima-Perú para evaluación de tratamiento ortodóncico. Su motivo de consulta fue "no me gusta mi sonrisa porque mis dientes están feos". Sus antecedentes médicos no mostraron ninguna contraindicación y refirió que su padre presenta sus mismas características faciales. En el análisis extraoral se registró un biotipo braquifacial, rostro simétrico, perfil facial cóncavo, mentón protruido y leve exposición de incisivos en sonrisa (Figura $1 \mathrm{~A}-\mathrm{C}$ ). Al examen clínico no se encontró desplazamiento mandibular entre relación céntrica y máxima intercuspidación, pero sí la presencia de un clic articular bilateral sin dolor en apertura y cierre.

Al examen clínico intraoral y análisis de modelos, se determinó una maloclusión de Clase III, mordida cruzada unilateral de canino e incisivo lateral superior izquierdo (OJ $-2,5 \mathrm{~mm})$, mordida bis a bis anterior de incisivos centrales superiores, apińamiento anterosuperior y anteroinferior de $-6 \mathrm{~mm}$ y $-4 \mathrm{~mm}$ respectivamente, discrepancia Bolton anterior por exceso inferior de 2,5 $\mathrm{mm}$, línea media dentaria superior desviada $1,5 \mathrm{~mm}$ a la izquierda y la inferior desviada $1,5 \mathrm{~mm}$ a la derecha (Figura 1 D-I, Figura 2).

En la radiografía panorámica se observó la presencia de cuatro terceras molares no erupcionadas, siendo las inferiores en posición horizontal (Figura 3). El análisis cefalométrico lateral reveló una relación esquelética de Clase III (ANB $-3^{\circ}$ ), patrón facial hipodivergente (GoGn-Sn $22^{\circ}$, FMA $19^{\circ}$ ), incisivo superior protruido y vestibularizado e inferior retruido y retroinclinado (Figura 4, Tabla). El índice de discrepancia del American Board Orthodontic fue de 27.

Tratamiento. Los objetivos de tratamiento fueron: (1) Mantener la relación esquelética Clase III, (2) Establecer la relación molar y canina Clase I, (3) Reposicionar incisivo superior en inferior, (4) Corregir la mordida cruzada anterior unilateral de canino e incisivo lateral superior izquierdo (5) Corregir la mordida bis a bis anterior y obtener overjet y overbite adecuado, (6) Eliminar el apiñamiento anterosuperior e inferior, (7) Corregir la línea media dental superior e inferior, (8) Establecer una relación oclusal funcional, (9) Mejorar el perfil del tercio inferior, y (10) Mejorar la exposición de los incisivos superiores en sonrisa.

Alternativas de tratamiento. Dado que el paciente se encuentra sin crecimiento se plantearon dos opciones de tratamiento. La primera opción fue un tratamiento combinado de ortodoncia y cirugía ortognática, con extracciones de primeras premolares superiores para la fase prequirúrgica; seguida de un avance maxilar con retroceso mandibular en la fase quirúrgica. 


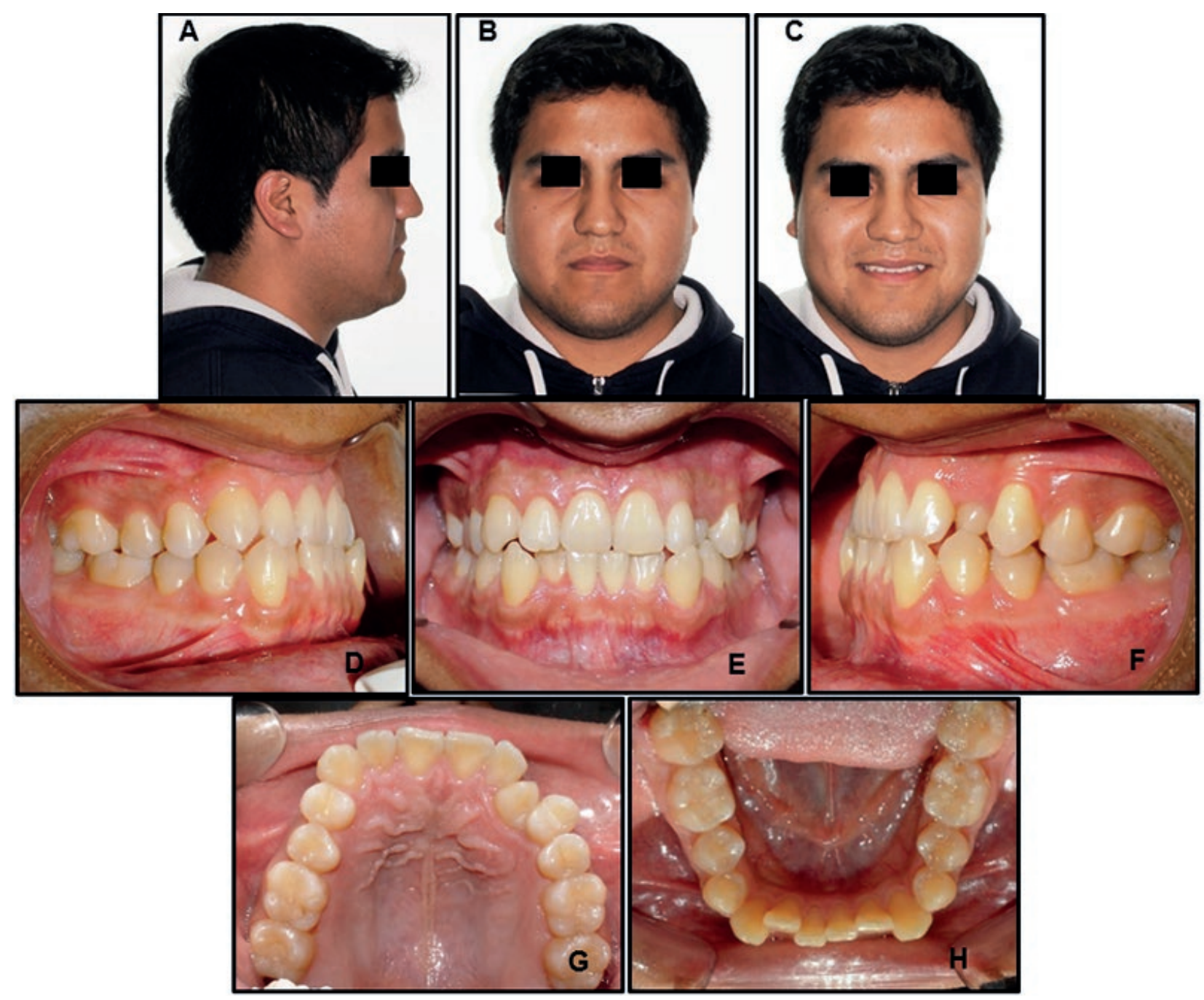

Figura 1. Fotografía de pretratamiento: A-C. Fotografías extraorales. D-H. Fotografías intraorales

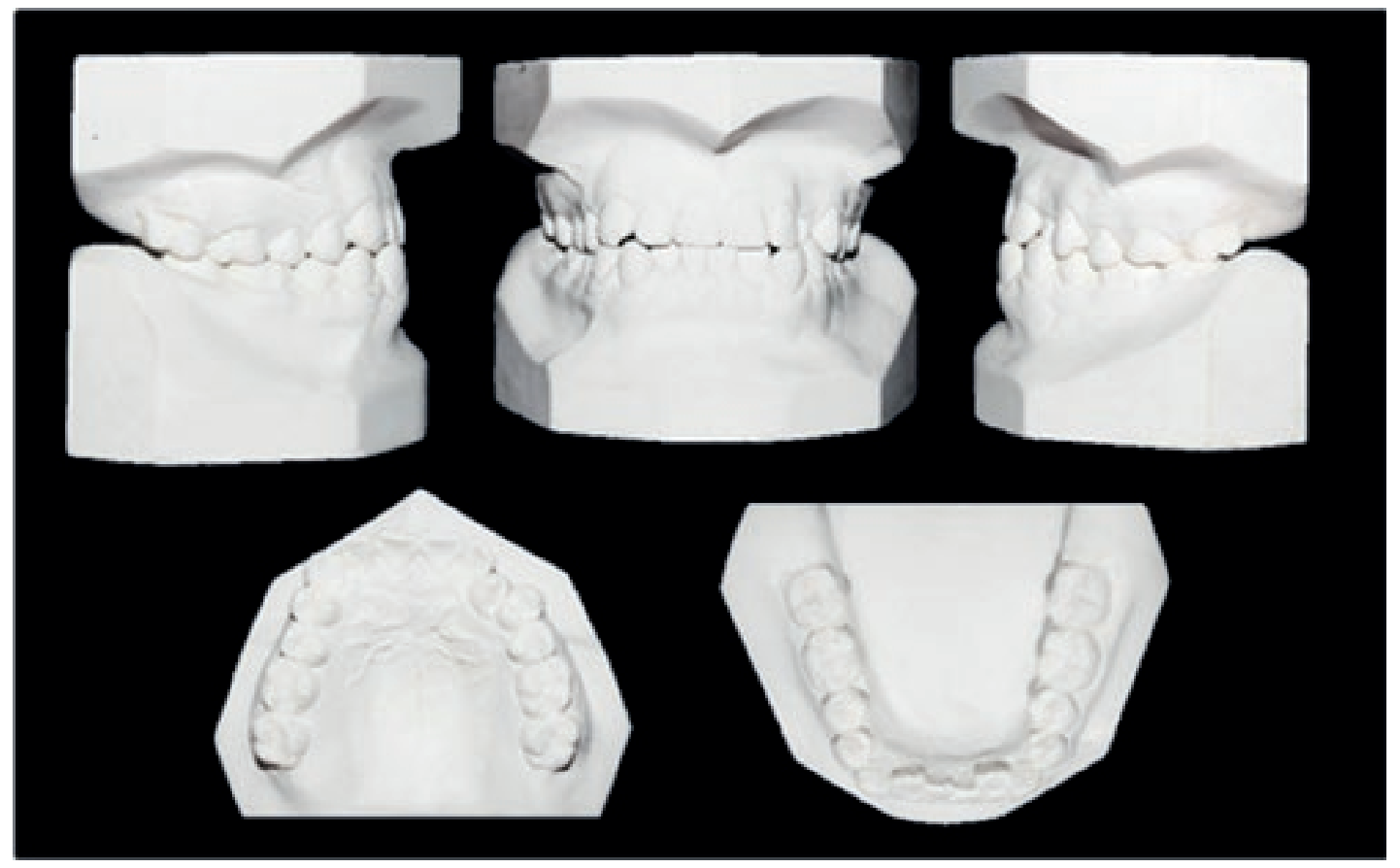

Figura 2. Modelos dentales pretratamiento 


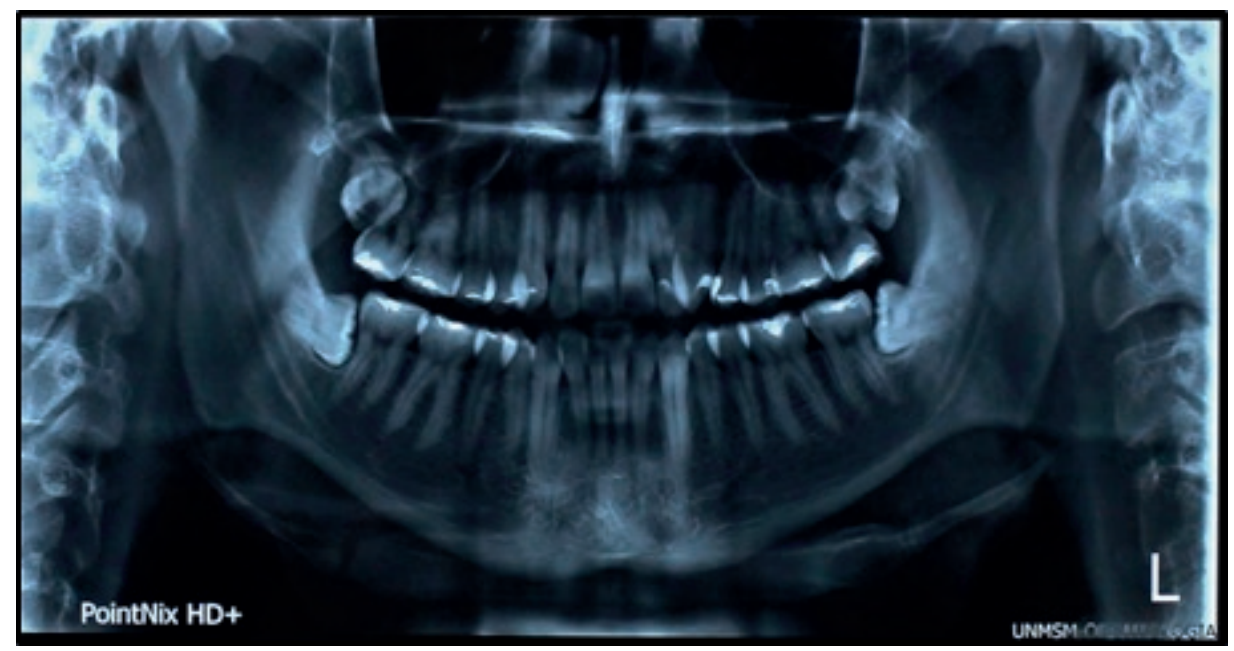

Figura 3. Radiografía panorámica pretratamiento

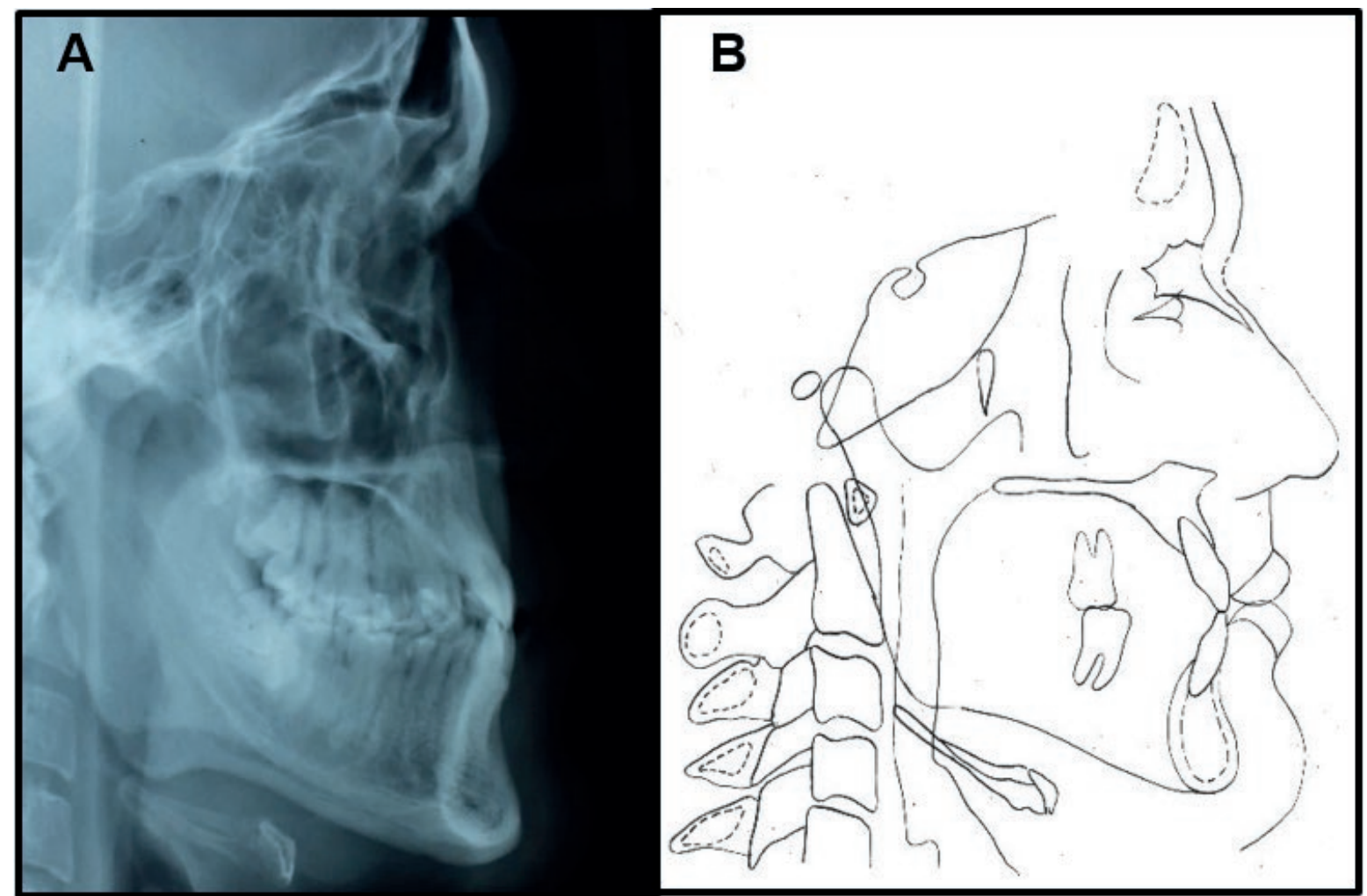

Figura 4. A. Radiografía cefalométrica pretratamiento; B. Trazado cefalométrico

La segunda opción fue un tratamiento ortodóncico de camuflaje utilizando las diferentes técnicas practicadas en la Clínica de Posgrado de Ortodoncia de la UNMSM: arcos segmentados ${ }^{15-18}$, arco multiloop ${ }^{9,19,20}$ y dobleces de compensación de primer, segundo y tercer orden $^{21}$.

El paciente declinó la opción quirúrgica porque no contaba con los medios económicos necesarios para solventarlo; adicionalmente, su patrón facial se encontraba dentro de los límites aceptados; de esta manera, la segunda opción fue escogida.

Progreso del tratamiento. Antes de iniciar el tratamiento ortodóncico, se indicó al paciente la extracción de sus terceros molares inferiores. Se instaló la aparatología superior MBT slot 0,022 ” x 0,028 ”, se confeccionó arcos segmentados posteriores con alambre de acero de 0019 " x 0,025" y un arco utilitario de protrusión de TMA 0,017 " x 0,025" que se activó modificando los escalones de modo que la sección anterior estuvo $2 \mathrm{~mm}$ por delante de los brackets de incisivos. Esto permitió la vestibularización de dichas piezas, así como la ganancia de espacio para la pieza 23 (Figura 5 A-C). Después de la reposición de incisivos superiores, se instaló dos bloques de acrílico posteriores para hacer el levante de mordida y corregir la mordida cruzada del canino superior, a su vez, se utilizaría como dispositivo de anclaje para evitar los efectos indeseados al uso de elásticos de Clase 
III. Con un cantilever logarítmico de TMA 0,017 ” $x$ 0,025 ” con una fuerza de $50 \mathrm{~g}$ se corrigió la rotación y descruzó la pieza 23 (Figura 5 D-E). Por otra parte, en el arco inferior se colocó la aparatología dos meses después de la superior, se instaló un arco multiloop fabricado con elgiloy azul 0,016 x 0,016" y con activaciones de tip back para corregir el tipping del sector posteroinferior sin ligar los incisivos; se indicó el uso de elásticos intermaxilares de Clase III cortos $3 / 16$ de 4,5 onzas (Figura 5 G-I). Después de tres meses, se decidió ligar los incisivos inferiores con arco continuo NiTi 0,014" hasta llegar a un arco de 0,016". Adicionalmente, se realizó strip- ping para eliminar el Bolton inferior de $2,5 \mathrm{~mm}$, lo que contribuyó a corregir el apińamiento y la línea media (Figura $5 \mathrm{M}-\mathrm{O}$ ). Mientras tanto, se corrigió la mordida cruzada de la pieza 23 a los 2 meses de instalado el cantilever logarítmico (Figura $5 \mathrm{~J}-\mathrm{L}$ ), se retiraron los bloques de acrílico posterior para colocar arcos continuos $\mathrm{NiTi}$ 0,016 " hasta llegar a un arco de acero 0,018” x 0,025", lo que permitió expresar el torque del bracket del canino $\left(-7^{\circ}\right)$ sin requerir torque individual (Figura $6 \mathrm{~A}-\mathrm{C}$ ). Se confeccionó un sliding jig usado con elásticos de Clase III para mesializar premolares y molares del segundo cuadrante (Figura 6 D-F).

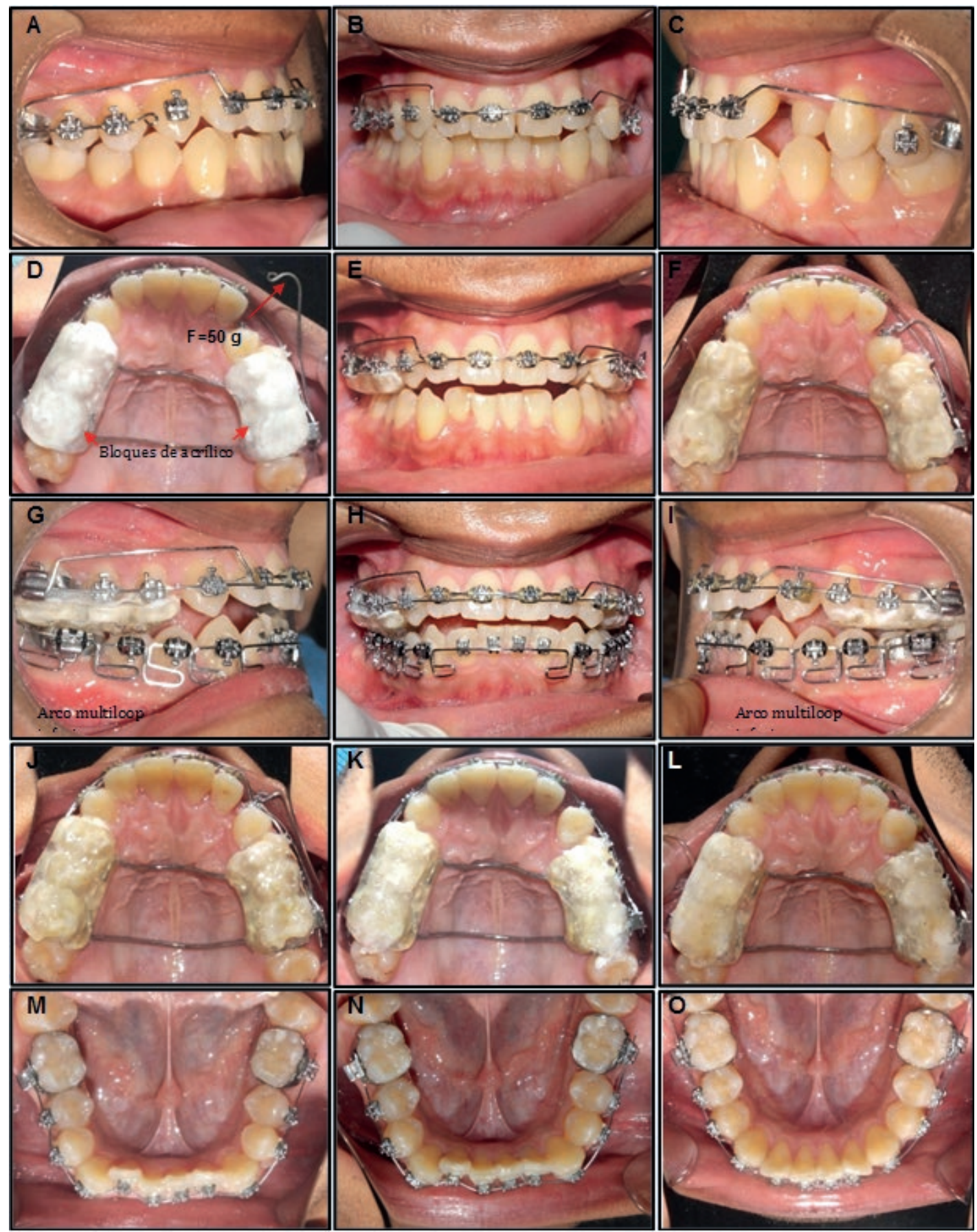

Figura 5. Fotografías intraorales durante el tratamiento: A-C. Alineamiento y nivelación tratamiento, arco de protrusión para vestibularización de incisivos. D-F. Activación de Cantiliver logarítmico F=50 g después de instalación de bloques de acrílico. G-I. Arco multiloop inferior con activaciones de tip back para corregir el tipping del sector posteroinferior. J-L. Desrotación de canino. M-O. Alineamiento del arco inferior 


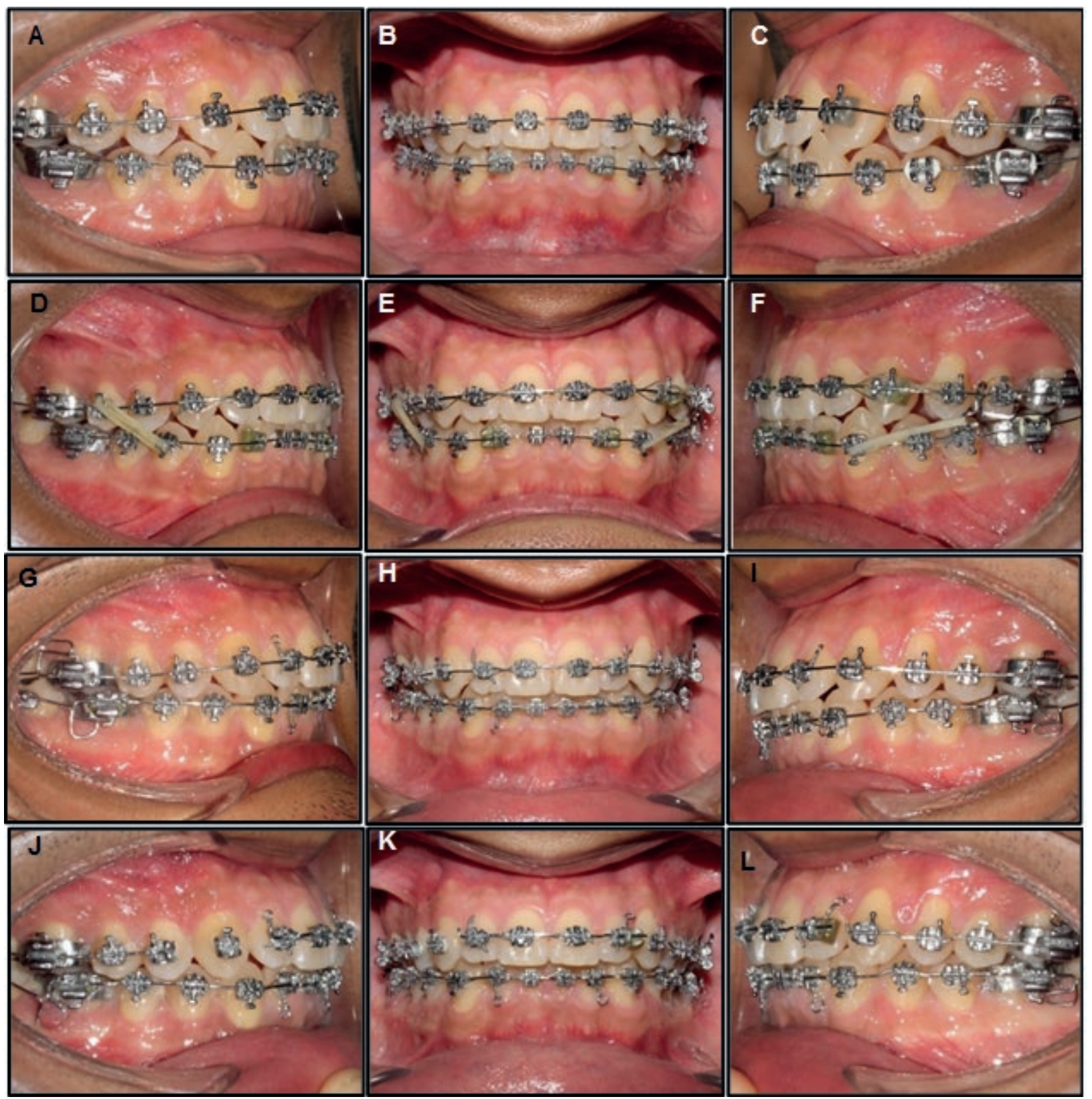

Figura 6. Fotografías intraorales durante el tratamiento: A-C. Nivelación de arcos superior e inferior. D-E. Uso de elásticos intermaxilares de Clase III cortos 3/16 de 4.5 onzas. F-I. Uso de sliding jig más elásticos de Clase III para mesializar premolares y molares superiores. G-I. Arcos rectangulares superior e inferior, véase los loop en bota a nivel de molares para mejora de la intercuspidación. J-L. Fragmentación de arco superior para mejora de asentamiento.

Para la etapa de finalización, se utilizaron arcos de acero 0,020 " en ambas arcadas con dobleces de primer, segundo orden; se continuó el uso de elásticos triangulares de Clase I 3/16 de 4,5 onzas y fragmentación de arco superior (Figura $6 \mathrm{G}-\mathrm{L}$ ),

El tiempo total de tratamiento fue de 2 ańos y 5 meses. Se instaló retenedor fijo en el arco inferior y un retenedor removible en el arco superior.

Resultados del tratamiento. Al análisis de modelos postratamiento, se determinó que los objetivos del tratamiento fueron alcanzados. Un aceptable overjet y overbite fueron obtenidos, así como una relación molar y canina de Clase I. El perfil del tercio inferior mejoró notablemente por rotación del mentón hacia abajo y atrás conjuntamente con el labio inferior. Se corrigió la mordida cruzada anterior unilateral de canino e incisivo lateral superior izquierdo, así como la línea media dental superior e inferior con una adecuada intercuspidación.

La sonrisa del paciente fue significativamente mejorada debido a que se produjeron movimiento de vestibularización y extrusión de los incisivos superiores con mayor exposición de éstos en sonrisa. El paciente no reportó dolor o disconformidad a nivel del ATM durante todo el tratamiento.

La radiografía panorámica final mostró un aceptable paralelismo entre las raíces de los dientes; no se visualizó signos de reabsorción radicular. En el análisis cefalométrico se observó una disminución del ángulo ANB de -03 a $-02^{\circ}$ y aumento del SN-MP de $22^{\circ}$ a $25^{\circ}$. El IMPA cambio de $92^{\circ}$ a $93^{\circ}$. El análisis de discrepancia 
de la American Board Orthodontics final fue de 6 (Figuras 7-11 y Tabla).

Se realizó un control a los 11 meses después del retiro de la aparatología (Figura 12) y se apreció la estabilidad de las posiciones y relaciones dentales intra e interarcos. Se comprobó también la estabilidad de las guías funcionales caninas y la guía anterior.

\section{Discusión}

Existe controversia aun sobre los límites a considerar si un caso de maloclusión de Clase III puede ser o no considerado para camuflar; actualmente no existe un consenso universal. Stellzig-Eisenhauer et al. buscaron identificar a través de un análisis discriminativo, variables dentoesqueletales para determinar si el desarrollo de una maloclusión de Clase III debe ser tratado con camuflaje o cirugía; determinaron que, de todas las variables encontradas, el índice de Wits fue el mejor factor discriminante. En este estudio, el promedio del índice de Wits para pacientes que fueron tratados con camuflaje fue de $-4,6+/-1,7 \mathrm{~mm}^{14,22}$. En el paciente, el índice de Wits fue de $-8 \mathrm{~mm}$, significativamente mayor al límite sugerido por el estudio para un tratamiento de camuflaje. Sin embargo, existen otras variables que se deberían tomar en cuenta para diferenciar si un caso es de camuflaje o para cirugía. Tseng et al. determinaron en su estudio seis parámetros cefalométricos como medición de necesidad de tratamiento quirúrgico en un paciente de Clase III, donde el paciente es recomendado a tener una cirugía ortognática por tratamiento si solo cuatro de las seis condiciones se cumplen ${ }^{1,23}$. Los parámetros fueron overjet $\leq-4,73 \mathrm{~mm}$, índice de Wits $\leq-11,18 \mathrm{~mm}$, $\mathrm{IMPA} \leq 80,8^{\circ}$, relación $\mathrm{Mx} / \mathrm{Mn} \leq 65,9 \%$, overbite $\leq$ $-0,18 \mathrm{~mm}$ y ángulo gonial $\geq 120,8^{\circ}$. En el paciente, cinco de seis parámetros de este estudio fueron cumplidos, lo cual indicaría ser un paciente para un tratamiento de camuflaje. Ambos estudios utilizaron radiografías cefalométricas para identificar las variables predictivas donde se encontró similitud de variables, pero diferencia en sus promedios; como por ejemplo, el índice de Wits, los promedios son diferentes entre ambos estudios.
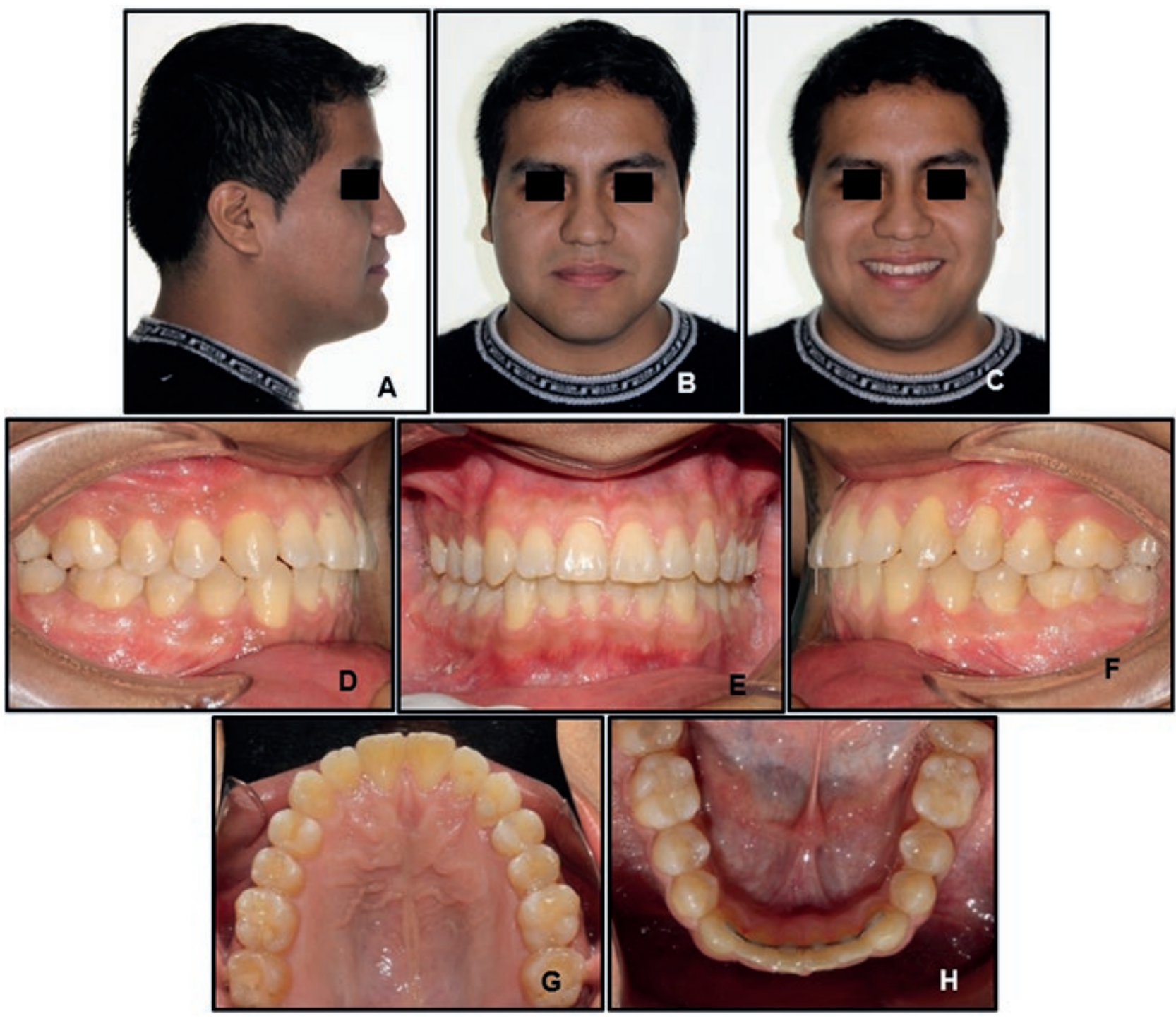

Figura 7. Fotografía intraorales postratamiento: A-C. Fotografías extraorales. D-H. Fotografías intraorales 


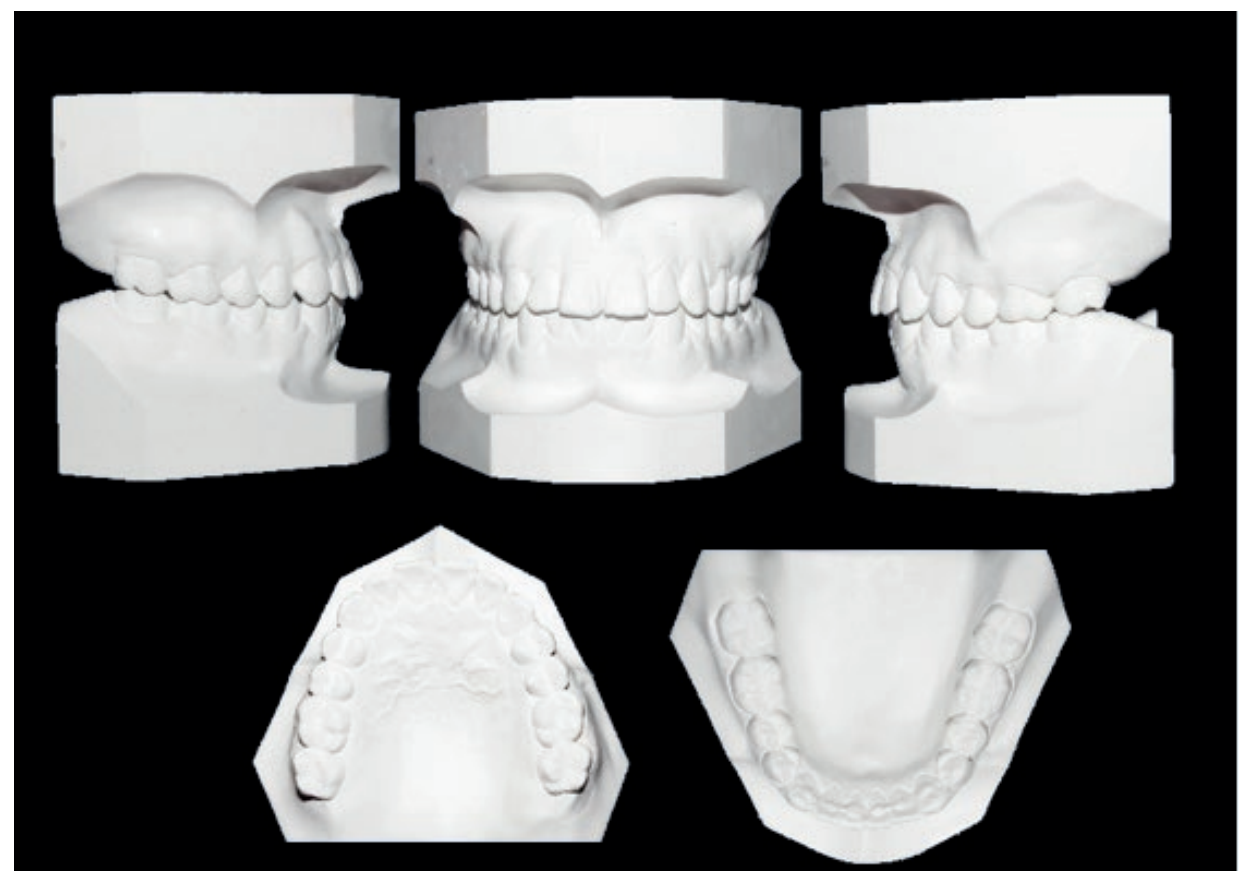

Figura 8. Modelos dentales postratamiento

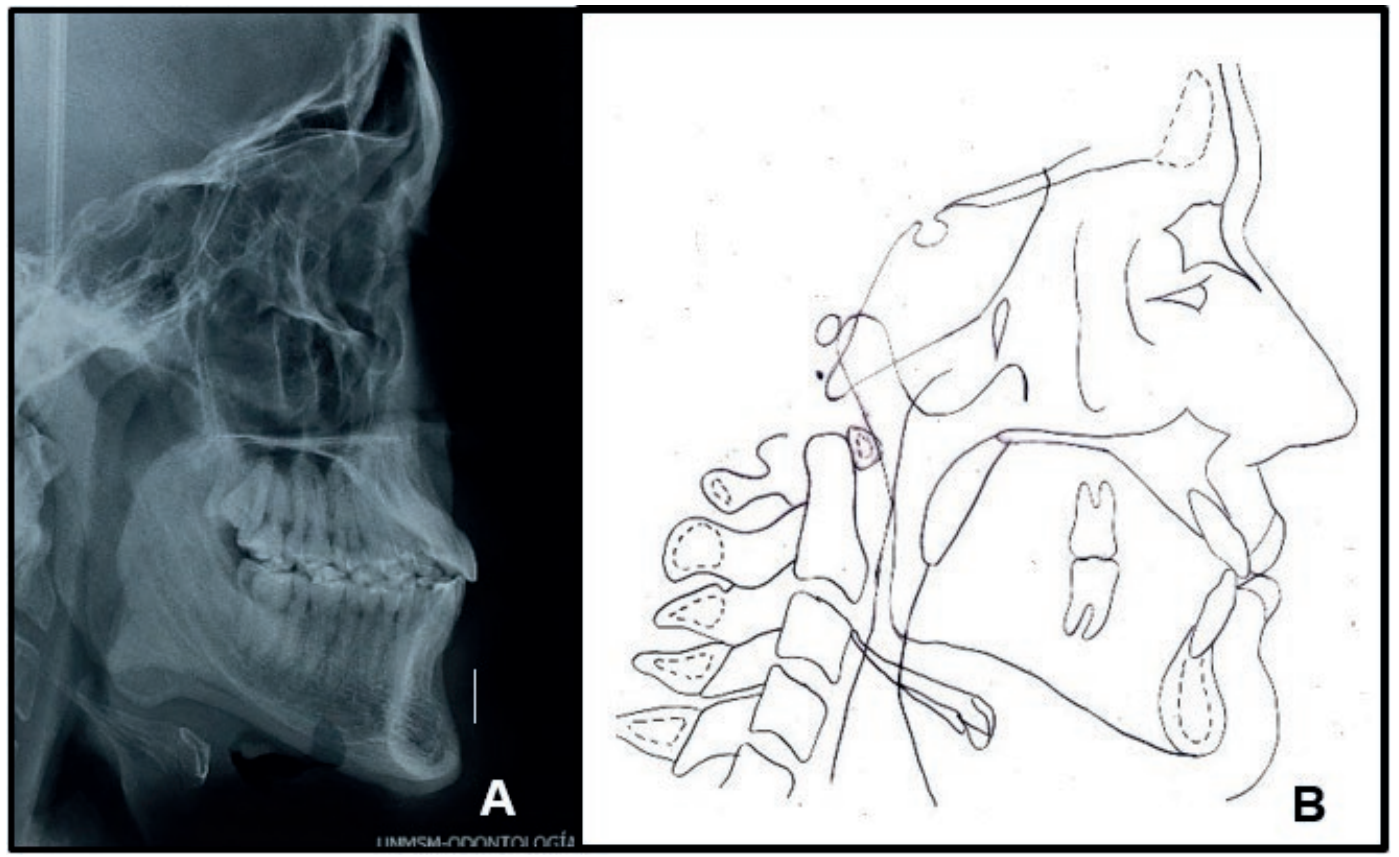

Figura 9. A. Radiografía cefalométrica postratamiento. B. Trazado cefalométrico postratamiento

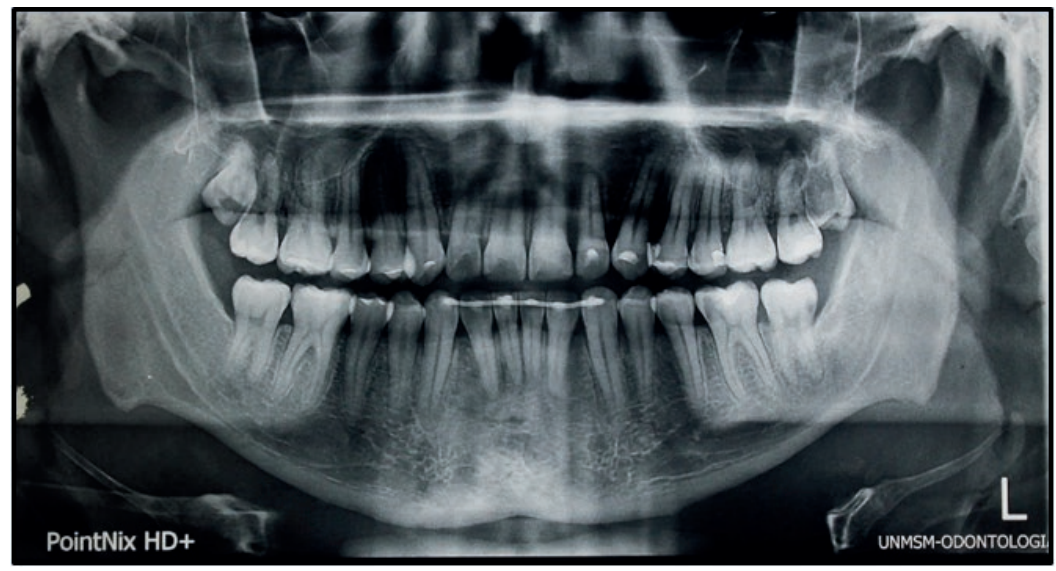

Figura 10. Radiografía panorámica postratamiento 


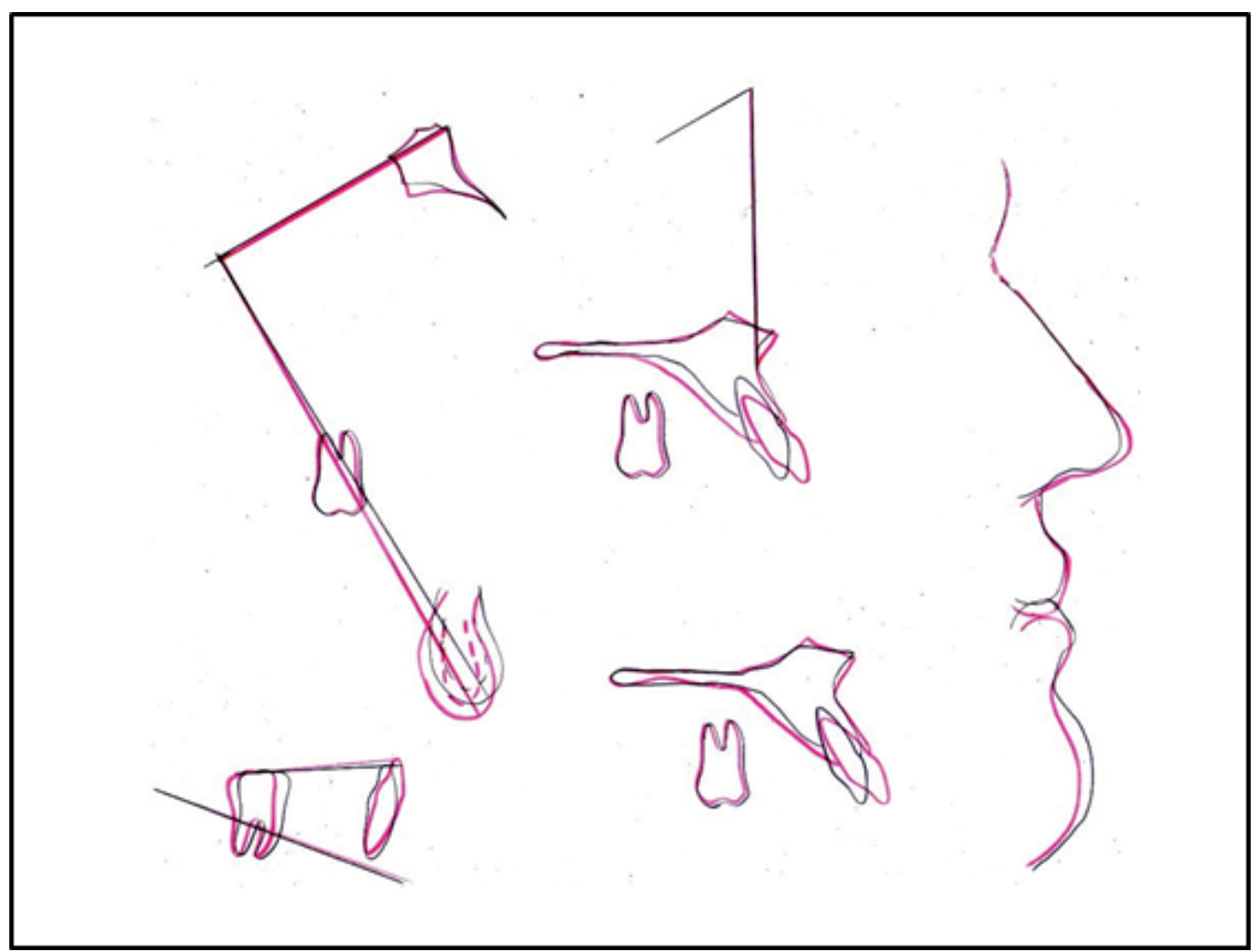

Figura 11. Superposiciones de trazados cefalométricos de pretratamiento (línea de color negro) y de postratamiento (línea de color rojo). Según los cinco campos de Ricketts
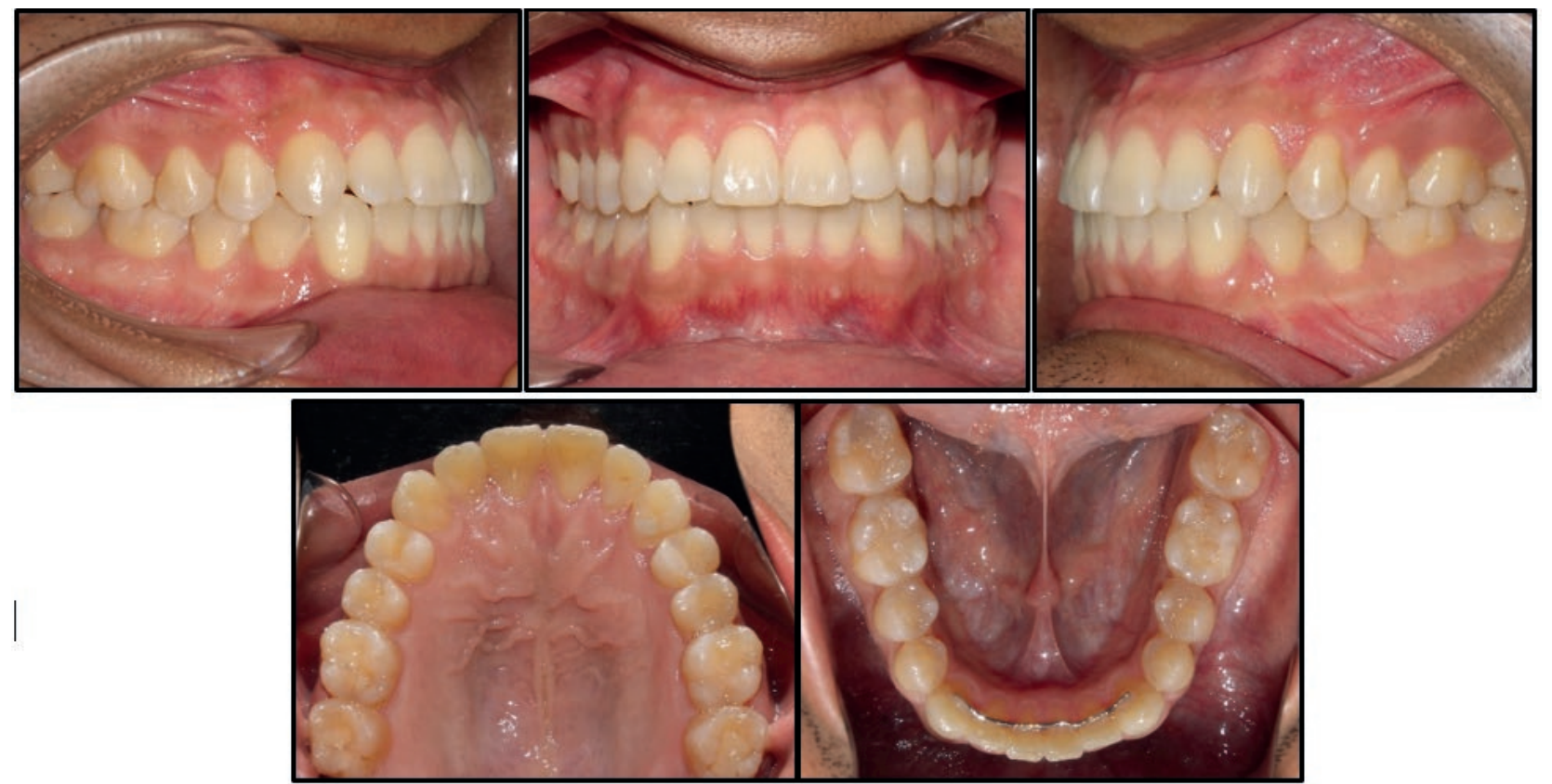

Figura 12. Fotografías intraorales de control 11 meses postratamiento

Moon et al. concluyeron que los pacientes de Clase III con un patrón esquelético más hipodivergente, generalmente responde mejor al tratamiento de camuflaje ${ }^{24}$. Franchi et al. en su estudio manifestaron que el ángulo palatino al plano mandibular, es un indicador predictivo de buenos resultados en el tratamiento temprano de Clase III ${ }^{25}$. Este paciente, presenta SN-PM: $22^{\circ}$ y PP$\mathrm{PM}: 18^{\circ}$; indicadores que serían afín a un tratamiento de camuflaje; además de presentar otras características favorables como un perfil facial con aceptable balance, segmentos bucales que son aproximados a una Clase I, ausencia de mordida abierta y desviación mandibular.

La reposición e inclinación de los incisivos en un camuflaje de Clase III tiene un grado de dificultad por su discrepancia esquelética. La excesiva inclinación vestibular de los incisivos repercute de manera negativa en una vista frontal ${ }^{26}$. En el paciente la inclinación del incisivo superior aumentó $5^{\circ}$ y $3,5 \mathrm{~mm}$ de reposición (Tabla), esta nueva inclinación no afectó en la exposición de incisivos en sonrisa al final de tratamiento. 
Tabla. Medidas cefalométricas pretratamiento y postratamiento

\begin{tabular}{lcc}
\hline & Pretratamiento & Postratamiento \\
\hline Ángulo SNA $\left(^{\circ}\right)$ & 82 & 82 \\
Ángulo SNB $\left(^{\circ}\right)$ & 85 & 84 \\
Ángulo ANB $\left(^{\circ}\right)$ & -03 & -02 \\
Ángulo SND $\left(^{\circ}\right)$ & 85 & 84 \\
1:NA (mm) & 6,5 & 10 \\
1:NA $\left(^{\circ}\right)$ & 28 & 33 \\
1:NB $(\mathrm{mm})$ & 1,5 & 3,5 \\
1:NB $\left(^{\circ}\right)$ & 23 & 22 \\
IMPA $\left(^{\circ}\right)$ & 92 & 93 \\
FMA $\left({ }^{\circ}\right)$ & 19 & 18 \\
Overjet(mm) & 0 & 3 \\
Overbite(mm) & 0 & 2,5 \\
Ocl:SN $\left({ }^{\circ}\right)$ & 10 & 8 \\
GoGn:SN $\left(^{\circ}\right)$ & 22 & 25 \\
Labio superior: línea S (mm) & $-3,5$ & -3 \\
Labio inferior: línea S (mm) & -3 & 2,5 \\
Eje Y $\left({ }^{\circ}\right)$ & 56 & 57 \\
Índice de Wits & -8 & -6 \\
Relación Mx/Mn & $69 \%$ & $69 \%$ \\
Ângulo gonial & 121 & 121 \\
\hline
\end{tabular}

Diferentes biomecánicas son utilizadas con arco continuo o arco segmentado, sin embargo, la técnica de arco segmentado (TAS), permite diseñar sistema de fuerzas con mejor determinación y control ${ }^{15,17}$. En las primeras fases del tratamiento de este caso se inició con la segmentación del arco superior para facilitar el uso de alambres flexibles en áreas de mayor movimiento, como lo sugiere la TAS ${ }^{15,16}$ y la bioprogresiva de Rickets ${ }^{18}$ donde se aprovecha los principios biomecánicos para conseguir el movimiento dentario deseado teniendo mejor control de los efectos adversos. El uso de arco utilitario, bloques de acrílico, cantilever, arco multiloop y arco continuo en diferentes etapas de tratamiento, permitieron manejar el caso con mayor predictibilidad.

Una de las principales preocupaciones de la estabilidad del tratamiento de camuflaje en maloclusiones de Clase III, es la posición final del incisivo, el cual depende del equilibrio muscular y funcional ${ }^{24}$. En el paciente no se registró recidiva en los once meses postratamiento. La estabilidad a mediano o largo plazo está asociada a una adecuada intercuspidación, un overjet de $2-3 \mathrm{~mm}$, overbite $3 \mathrm{~mm}$, raíces paralelas y dentro de su hueso basal, entre otros; sin embargo, existen casos de camuflaje severos donde se recomienda el uso de retenedores fijos permanente ${ }^{24}$.

Se concluye que el tratamiento de camuflaje de Clase III es una opción factible en pacientes adultos que rechazan la opción quirúrgica; sin embargo, son realizadas en casos de ligera a moderada complejidad con una estética facial aceptable.
Actualmente no existe un consenso universal sobre los límites entre el camuflaje ortodóncico y cirugía ortognática de una maloclusión de Clase III.

El uso de bloques de acrílico posterior, arco utilitario de protrusión, arco multiloop y uso de elásticos intermaxilares pueden ser utilizados para el tratamiento de camuflaje de Clase III.

Los resultados de tratamiento de camuflaje fueron estables en los 11 meses de control postratamiento.

\section{Referencias bibliográficas}

1. Tseng YC, Pan CY, Chou ST, Liao CY, Lai ST, Chen $\mathrm{CM}$, et al. Treatment of adult Class III malocclusions with orthodontic therapy or orthognathic surgery: Receiver operating characteristic analysis. Am J Orthod Dentofacial Orthop. 2011;139:485-93.

2. Farret MM, Benitez Farret MM, Farret AM. Orthodontic camouflage of skeletal Class III malocclusion with miniplate: a case report. Dent Press J Orthod. 2016;21(4):89-98.

3. Troy BA, Shanker S, Fields HW, Vig K, Johnston W. Comparison of incisor inclination in patients with Class III malocclusion treated with orthognathic surgery or orthodontic camouflage. Am J Orthod Dentofacial Orthop. 2009;135(2):146.e1-9.

4. Hardy DK, Cubas YP, Orellana MF. Prevalence of angle class III malocclusion: A systematic review and meta-analysis. Open J Epidemiol. 2012;2:75-82.

5. Martinez P, Bellot-Arcís C, Llamas JM, Cibrian R, Gandia JL, Paredes-Gallardo V. Orthodontic camouflage versus orthognathic surgery for class III deformity: comparative cephalometric analysis. Int J Oral Maxillofac Surg.2017;46(4):490-5.

6. Bakr A, Rabie M, Wong RWK, Min GU. Treatment in Borderline Class III Malocclusion:Orthodontic Camouflage (Extraction) Versus Orthognathic Surgery. Open Dent J. 2008;2:38-48.

7. Burns NR, Musich DR, Martin C, Razmus T, Gunel E, Ngan P. Class III camouflage treatment: What are the limits? Am J Orthod Dentofacial Orthop. 2010;137(1):9. e1-13.

8. Baccetti T, Reyes BC, McNamara JA. Gender differences in Class III malocclusion. Angle Orthod. 2005;75(4):510-20.

9. Marañón-Vásquez GA, Soldevilla Galarza LC, Tolentino Solis FA, Wilson C, Romano FL. Aesthetic and functional outcomes using a multiloop edgewise archwire for camouflage orthodontic treatment of a severe Class III open bite malocclusion. J Orthod. 2017;44(3):199-208.

10. Bailey LJ, Haltiwanger LH, Blakey GH, Proffit WR. Who seeks surgical-orthodontic treatment: a current review. Int J Adult Orthodon Orthognath Surg. 2001;16(4):280-92.

11. Ying Y, Han X, Guo Y, Li J, Bai D. Nonsurgical correction of a Class III malocclusion in an adult by miniscrew-assisted mandibular dentition distalization. Am J Orthod Dentofac Orthop. 2013;143:877-87. 
12. Valladares JN. Compensatory orthodontic treatment of skeletal Class III malocclusion with anterior crossbite. Dent Press J Orthod. 2014;19(1):113-22.

13. Kerr WJ, Miller S, Dawber JE. Class III malocclusion: surgery or orthodontics?. Br J Orthod 1992;19:21-24.

14. Stellzig-Eisenhauer A, Lux CJ, Schuster G. Treatment decision in adult patients with Class III malocclusion: Orthodontic therapy or orthognathic surgery?. Am J Orthod Dentofacial Orthop. 2002;122(1):27-37.

15. Burstone, C.J. The rationale of the segmented arch. Am J Orthod Dentofacial Orthop. 1962;47(11):805-21.

16. Burstone C. The Mechanics Of The Segmented Arch Tecniques. Angle Orthod. 1966;36(2):99-120.

17. Sakima M. Técnica do Arco Segmentado de Burstone. Dental Press Ortodon Facial. 2000;(2):91-115.

18. Ricketts, RM. Técnica bioprogresiva de Ricketts. Buenos Aires. Editorial Médica Panamericana; 1999.

19. Kim YH. Anterior openbite and its treatment with multiloop edgewise archwire. Angle Orthod. 1987;57:290-321.

20. Sato S, Takamoto K, Suzuki Y. Posterior discrepancy and development of skeletal class III malocclusion: its im- portance in orthodontic correction of skeletal Class III malocclusion. Orthod. Orthod Rev. 1987;2(6):16-29.

21. Tweed, Ch. The aplication Principle of the Edgewise Arch in the tretment of Malocclusion. Angle Orthod.1941;11(1):5-11.

22. Jacobson A. The "Wits" appraisal of jaw disharmony. Am J Orthod Dentofacial Orthop. 2003;124:470-9.

23. Tseng LLY, Chang CH, Roberts WE. Diagnosis and conservative treatment of skeletal Class III malocclusion with anterior crossbite and asymmetric maxillary crowding. Am J Orthod Dentofacial Orthop. 2016;149(4):555-66.

24. Moon YM, Ahn SJ, Chang YI. Cephalometric predictors of longterm stability in the early treatment of Class III malocclusion. Angle Orthod. 2005;75:747-53.

25. Franchi L, Baccetti T, Tollaro I. Predictive variables for the outcome of early functional treatment of Class III malocclusion. Am J Orthod Dentofacial Orthop. 1997;112:80-6.

26. Park JH, Yu J, Bullen R. Camouflage treatment of skeletal Class III malocclusion with conventional orthodontic therapy. Am J Orthod Dentofacial Orthop. 2017;151(4):804-11. 
